\title{
ON SEMILINEAR FRACTIONAL ORDER DIFFERENTIAL INCLUSIONS IN BANACH SPACES
}

\begin{abstract}
We are considering the Cauchy problem for a semilinear fractional differential inclusion in a Banach space. By using the fixed point theory for condensing multivalued maps, we prove the local and global theorems of the existence of mild solutions to this problem. We verify the compactness of the solutions set and its continuous dependence on parameters and initial data. We demonstrate also the application of the averaging principle to the investigation of the continuous dependence of the solutions set on a parameter in the case when the right-hand side of the inclusion is rapidly oscillating.

Key Words and Phrases: Fractional differential inclusion, semilinear differential inclusion, Cauchy problem, continuous dependence of solutions, averaging principle, fixed point, multivalued map, condensing map, measure of noncompactness.
\end{abstract}

2010 Mathematics Subject Classification: 34A60, 34C29, 34G25, 34K37, 47H04, 47H08, 47H10.

Acknowledgment. The work on the paper was carried out during Professor M. Kamenskii and Professor V. Obukhovskii's visit to the Department of Applied Mathematics, National Sun Yat-Sen University, and the Center of Fundamental Science, Kaohsiung Medical University, Kaohsiung, Taiwan, in 2015. They would like to express their gratitude to the members of the Department of Applied Mathematics and the Center of Fundamental Science for their kind hospitality. The work is supported by the Taiwan-Russia NNS-RFBR grant 14-01-92004, the research of the first, second and the third authors is supported by the RFBR grants 14-01-00468, 16-01-00386 and the work of the second author is supported by the Russian Science Foundation grant 14-21-00066 accomplished in the Voronezh State University. 


\section{REFERENCES}

[1] S. Abbas, M. Benchohra, G.M. N'Guerekata, Topics in Fractional Differential Equations, Developments in Mathematics, Springer, New York, 2012.

[2] R.R. Ahmerov, M.I. Kamenskii, A.S. Potapov, A.E. Rodkina, B.N. Sadowskii, Measures of Noncompactness and Condensing Operators, Birkhäuser, Boston-Basel-Berlin, 1992.

[3] D. Baleanu, K. Diethelm, E. Scalas, J.J. Trujillo, Fractional Calculus Models and Numerical Methods, World Scientific Publishing, New York, 2012.

[4] Yu.G. Borisovich, B.D. Gelman, A.D. Myshkis, V.V. Obukhovskii, Introduction to the Theory of Multivalued Maps and Differential Inclusions, Librokom, Moscow, 2011. (in Russian)

[5] J. Diestel, W.M. Ruess, W. Schachermayer, Weak compactness in $L^{1}(\mu, X)$, Proc. Amer. Math. Soc., 118(1993), 447-453.

[6] K. Diethelm, The Analysis of Fractional Differential Equations, Springer-Verlag, Berlin, 2010.

[7] R. Hilfer, Applications of Fractional Calculus in Physics, World Scientific, Singapore, 2000.

[8] M. Kamenskii, V. Obukhovskii, P. Zecca, Condensing Multivalued Maps and Semilinear Differential Inclusions in Banach Spaces, de Gruyter Series in Nonlinear Anal. Appl., 7, Walter de Gruyter, Berlin-New-York, 2001.

[9] T.D. Ke, V. Obukhovskii, N.C. Wong, J.C. Yao, On a class of fractional order differential inclusions with infinite delays, Applicable Anal., 92(2013), no. 1, 115-137.

[10] A.A. Kilbas, H.M. Srivastava, J.J. Trujillo, Theory and Applications of Fractional Differential Equations, North-Holland Mathematics Studies, 204, Elsevier Science B.V., Amsterdam, 2006.

[11] V. Lakshmikantham, Theory of fractional functional differential equations, Nonlinear Anal., 69(2008), no. 10, 3337-3343.

[12] V. Lakshmikantham, A.S. Vatsala, Basic theory of fractional differential equations, Nonlinear Anal., 69(2008), no. 8, 2677-2682.

[13] K.S. Miller, B. Ross, An Introduction to the Fractional Calculus and Fractional Differential Equations, John Wiley, Inc., New York, 1993.

[14] V. Obukhovskii, J.C. Yao, Some existence results for fractional functional differential equations, Fixed Point Theory, 11(2010), no. 1, 85-96.

[15] I. Podlubny, Fractional Differential Equations, Academic Press, San Diego, 1999.

[16] S.G. Samko, A.A. Kilbas, O.I. Marichev, Fractional Integrals and Derivatives, Theory and Applications, Gordon and Breach Sci. Publishers, Yverdon, 1993.

[17] V.E. Tarasov, Fractional Dynamics. Applications of Fractional Calculus to Dynamics of Particles, Fields and Media, Nonlinear Physical Science, Springer, Heidelberg, Higher Education Press, Beijing, 2010.

[18] Z. Zhang, B. Liu, Existence of mild solutions for fractional evolution equations, Fixed Point Theory, 15(2014), no. 1, 325-334.

Received: February 21, 2015; Accepted: October 10, 2015. 
